\title{
Prognostic Factors in Hospitalized COVID-19 Patients at the Start of the Pandemic in Colombia: A Retrospective Cohort Study
}

Jose A. Nuñez-Ramos ${ }^{1,2}$, Elias Forero Illera 1, 3 , Jorge Luis Quintero Barrios 1, 4 , Hugo Andres Macareno Arroyo 1,2 , Didier Johanna Larios Sanjuan ${ }^{1,2}$, Maria Carolina Manzur Barbur ${ }^{1,2}$, Ana Milena Mejia Sanjuanelo ${ }^{1,2}$, Mauro Duvan Mendoza Quevedo ${ }^{1,2}$, Diego Fernando Viasus-Perez 1,5

1. Health Sciences Division, Universidad del Norte, Barranquilla, COL 2. Internal Medicine, Hospital Universidad del Norte, Barranquilla, COL 3. Rheumatology, Hospital Universidad del Norte, Barranquilla, COL 4. Pulmonology, Hospital Universidad del Norte, Barranquilla, COL 5. Infectious Diseases, Hospital Universidad del Norte, Barranquilla, COL

Corresponding author: Jose A. Nuñez-Ramos, anunezj@uninorte.edu.co

\section{Abstract \\ Introduction}

Different factors are critical when assessing COVID-19 mortality, and can explain why severity differs so widely among populations. However, there is little information regarding prognostic factors and mortality in COVID-19 from Latin American countries.

\section{Objectives}

To determine prognostic factors in hospitalized COVID-19 patients and to evaluate the impact of tocilizumab use in patients with hyperinflammatory syndrome and severe disease defined by the National Early Warning Score 2 (NEWS2) with a value greater than or equal to seven points.

\section{Materials and methods}

This retrospective cohort study included hospitalized COVID-19 patients from May to July 2020. A multivariate logistic regression analysis was performed to determine independent factors associated with mortality.

\section{Results}

A total of 136 patients required hospital admission. In-hospital mortality was 39.7\%. Mortality was observed to be potentiated by older age, LDH serum levels and the presence of type 2 diabetes mellitus. Lymphopenia and lower PaO2/FiO2 ratio were more common in these patients. Similarly, patients who died were classified more frequently with severe disease. The independent factors associated with in-hospital mortality were age greater than 65 years, type 2 diabetes mellitus, NEWS2 greater than or equal to seven points and LDH greater than 400U/L. The use of Tocilizumab alone was not related with decreased in-hospital mortality.

Review began 04/21/2021 Review ended 04/27/2021 Published 05/06/2021

\section{() Copyright 2021}

Nuñez-Ramos et al. This is an open access article distributed under the terms of the Creative Commons Attribution License CC-BY 4.0., which permits unrestricted use, distribution, and reproduction in any medium, provided the original author and source are credited.

\section{Conclusions}

COVID-19 mortality in hospitalized patients was high and mainly related with older age, comorbidities, LDH and the severity of disease at hospital admission.

Categories: Internal Medicine, Infectious Disease

Keywords: covid-19, sars cov-2, covid prognosis, disease mortality, tocilizumab

\section{Introduction}

The coronavirus disease 2019 (COVID-19) was classified as a pandemic in February 2020 [1]. Approximately $5 \%-10 \%$ of patients require hospital admission due to the development of moderate to severe disease. Importantly, critically ill patients have higher mortality ranging from $20 \%$ to $60 \%$ [2]. Different factors are critical when assessing COVID-19 mortality, and these factors can explain why severity differs so widely among people and countries [3]. The risk of mortality is higher in older populations and in patients with comorbid conditions [4]. In addition, it has been proposed that an inadequate inflammatory response is one of the main causes of mortality in COVID-19 patients, leading to an important interest in order to identify treatments that can modulate this augmented response [5].

Dexamethasone has demonstrated a clinical impact reducing mortality in hypoxemic patients that require hospital admission [6]. Moreover, interleukin 6 (IL-6) receptor blockade seems to be a promising treatment 
for a specific group of COVID-19 patients. However, study findings are controversial [7-12].

There is little information regarding mortality and prognostic factors in COVID-19 from Latin América. Thus, this study aimed to determine prognostic factors in Colombian hospitalized COVID-19 patients and to evaluate the impact of tocilizumab use in patients with hyperinflammatory syndrome and severe disease defined by the National Early Warning Score 2 (NEWS2) greater than or equal to seven points.

\section{Materials And Methods}

This is a retrospective cohort study of hospitalized COVID-19 patients admitted, from May 20th to July 31st in a convenience sample, to the Hospital Universidad del Norte, a 136-bed university hospital in Soledad, Colombia. Clinical-suspected and laboratory-confirmed COVID-19 patients were included. A clinicalsuspected COVID-19 case was defined as a patient with upper or lower respiratory tract symptoms with compatible radiological computerized tomography (CT) lung scan (peripheral ground-glass opacities) and no other etiologies documented. Laboratory-confirmed COVID-19 case was defined as a clinical-suspected COVID-19 case with positive results in real-time polymerase chain reaction (RT-PCR) for severe acute respiratory syndrome coronavirus 2 (SARS-CoV-2). RT-PCR for SARS-CoV-2 was performed from respiratory samples in all patients. The study was approved by the Institutional Ethics Committee (ID213-2020).

Patients were divided into two groups: (i) patients who survived; and (ii) patients who died. The independent variables were clinical characteristics and laboratory values. The treatment of COVID-19 pneumonia was according to institutional protocol and included antibiotics, oxygen, steroids and tocilizumab. Regarding tocilizumab, it was used in patients with hypoxemia and elevated markers of inflammation. Tocilizumab was not administered to patients with suspicion of concomitant bacterial infection. The administered intravenous dose was $400 \mathrm{mg}$ for individuals less than $75 \mathrm{~kg}$ and $600 \mathrm{mg}$ for those greater than or equal to 75 $\mathrm{kg}$. All tocilizumab doses were given within the first 24 hours of hospital admission.

To address illness severity we used the NEWS2 [13]. Severe disease was defined as NEWS2 score of greater than or equal to seven points. Hyperinflammation was defined according to recent validated criteria in COVID-19 infection [5] that included two or more of these laboratory findings: ferritin $>700 \mathrm{ng} / \mathrm{dl}$, c-reactive protein $>80 \mathrm{mg} / \mathrm{dl}$, d-dimer $>1,000 \mathrm{ug} / \mathrm{L}$, lymphocyte $<900$ cells/ul, or lactate dehydrogenase $(\mathrm{LDH})>400 \mathrm{U} / \mathrm{L}$.

\section{Statistical analysis}

The categorical variables were reported as frequencies and percentages and the continuous variables as median and interquartile range (IQR). Normal distribution was tested. The Mann-Whitney U-test was used for comparing continuous variables and chi-square or Fisher's test for qualitative variables. To determine independent factors associated with in-hospital mortality, a multivariate analysis was performed. The model included important clinical variables, including tocilizumab, and those with significant difference between groups. Results of multivariate analyses were reported as odds ratio (OR) and 95\% confidence intervals (CI). The goodness of fit of the multivariate model was assessed by the Hosmer-Lemeshow test and the accuracy by the area under receiver operating characteristic (AUROC) curve. We performed subgroup analyses according to the presence of hyperinflammation and severe disease using NEWS $2 \geqslant 7$. The threshold for statistical significance was defined as a two-tailed $\mathrm{p}<0.05$. All analyses were performed using the SPSS software, version 25 (IBM Corp., Armonk, NY).

\section{Results}

During the study period, 136 patients with COVID-19 were admitted to the hospital. Regarding RT-PCR results, 113 (83.1\%) were classified as laboratory-confirmed COVID-19 and 21 (16.9\%) as clinical-suspected COVID-19.

\section{Characteristics of the cohort}

Clinical features and laboratory findings of all patients are shown in Table 1. Among the total population, 59 (43.4\%) patients were older than 65 years and were mostly males. The main comorbid condition was hypertension, followed by type 2 diabetes mellitus. The most common symptoms were cough, fever and dyspnea. Diarrhea and gastrointestinal manifestations were present in $10 \%$ of patients. Most patients had altered laboratory findings, such as lymphopenia, elevated ferritin, LDH, C-reactive protein and d-dimer. Regarding lung CT-scan, $90 \%$ of patients were classified as having typical findings of SARS-CoV2 pneumonia (CO-RADS 4 and 5). Once admitted, 41 (30.1\%) received antiviral therapy (hydroxychloroquine, oseltamivir, or lopinavir/ritonavir). Remdesivir was not an option considering it is not available in Colombia. Regarding interventions, 61 patients (44.9\%) received tocilizumab and 85 (66.4\%) corticosteroids (methylprednisolone or dexamethasone). In-hospital mortality was $39.7 \%$, accounting for 54 patients. Ninety (66.2\%) patients required ICU admission and almost $10 \%$ were intubated at emergency admission. 


\section{Cureus}

\begin{tabular}{|c|c|c|c|c|}
\hline Clinical features & All patients, $n=136$ & Survivors, $\mathrm{n}=82$ & Non-survivors, $n=54$ & p-value \\
\hline Age, years & $62(51-74)$ & $57(45-66)$ & $68(60-79)$ & $<0.001$ \\
\hline $18-40$ years & $18(13.2)$ & $16(19.5)$ & $2(3.7)$ & \\
\hline 41-65 years & $60(44.1)$ & $44(53.7)$ & $16(29.6)$ & \\
\hline$>65$ years & $58(42.6)$ & $22(26.8)$ & $37(68.5)$ & $<0.001$ \\
\hline Male & $73(53.7)$ & $46(56.1)$ & $27(50)$ & 0.485 \\
\hline Hypertension & $55(40.4)$ & $29(35.4)$ & $26(48.1)$ & 0.137 \\
\hline Type 2 diabetes & $42(30.9)$ & $20(24.4)$ & $22(40.7)$ & 0.001 \\
\hline Cardiovascular disease & $4(2.9)$ & $1(1.2)$ & $3(5.6)$ & 0,301 \\
\hline COPD/asthma & 11 (8) & $4(4.8)$ & $7(12.9)$ & 0,82 \\
\hline Days of symptoms & $7(4-8)$ & $7(5-9)$ & $4(3-7)$ & 0.023 \\
\hline Presence of fever & $100(73.5)$ & $66(80.5)$ & $34(63)$ & 0.978 \\
\hline Cough & $93(68.4)$ & $56(68.3)$ & $37(68.5)$ & 0.354 \\
\hline Dyspnea & $101(74.3 \%)$ & $52(85.2)$ & $49(65.3)$ & 0,008 \\
\hline Diarrhea & $17(12.5)$ & $9(14.8)$ & $8(10.7)$ & 0.473 \\
\hline \multicolumn{5}{|l|}{ Admission laboratory findings } \\
\hline $\mathrm{PaO} 2 / \mathrm{FiO} 2$ ratio & $203(110-338)$ & $247(155-360)$ & $158(51-283)$ & 0.001 \\
\hline D-dimer level (ug/L) & $700(300-2150)$ & $660(300-1400)$ & $980(400-6500)$ & 0,037 \\
\hline Ferritin level (ng/dl) & $1058(464-1650)$ & 1051 (485-1643) & 1144 (457-1650) & 0.870 \\
\hline LDH level (u/L) & $411(330-560)$ & $380(288-433)$ & $549(410-662)$ & $<0.001$ \\
\hline C-reactive protein level (mg/dl) & $161(125-174)$ & $159(104-177)$ & $162(140-177)$ & 0.463 \\
\hline Lymphocytes (cells/ul) & $810(552-1220)$ & $965(607-1300)$ & $690(487-1115)$ & 0.035 \\
\hline Aspartate aminotransferase (U/L) & $50(36-77)$ & $45(31-72)$ & $56(38-80)$ & 0.108 \\
\hline Alanine aminotransferase (U/L) & $40(25-66)$ & $40(27-71)$ & $43(24-61)$ & 0.469 \\
\hline Creatinine (mg/dl) & $1.1(0.9-1.5)$ & $1.0(0.9-1.3)$ & $1.4(1.0-2.2)$ & $<0.001$ \\
\hline NEWS2 score & $6(4-8)$ & $6(4-7)$ & $8(5-10)$ & $<0.001$ \\
\hline Nasal cannula & $40(29.4 \%)$ & $16(26.2)$ & $24(32)$ & 0.463 \\
\hline Non-rebreather mask & $51(37.5 \%)$ & $26(31.7)$ & $25(46.3)$ & 0.086 \\
\hline Orotracheal intubation at admission & $13(9.6)$ & $2(2.4)$ & $11(20.4)$ & 0.001 \\
\hline \multicolumn{5}{|l|}{ Interventions } \\
\hline Antivirals & $41(30.1 \%)$ & $23(28)$ & $18(33.3)$ & 0.534 \\
\hline Corticosteroids & $85(66.4)$ & $50(64.9)$ & $35(68.6)$ & 0.665 \\
\hline Tocilizumab & $61(44.9)$ & $40(48.8)$ & $21(38.9)$ & 0.256 \\
\hline ICU admission & $90(66.2)$ & $41(50)$ & $49(90.7)$ & 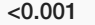 \\
\hline
\end{tabular}

TABLE 1: Baseline characteristics and according to survival in hospitalized COVID-19 patients. Values are median and interquartile range for continuous variables. Frequencies in \#(\%) for categorical. NEWS2: National Early Warning Score 2; LDH: lactate dehydrogenase; COPD: chronic obstructive pulmonary disease; ICU: intensive care unit. 


\section{Cureus}

\section{Factors associated with mortality}

Patients who survived were compared with patients who died (Table 1). Patients who died were older and had more frequently type 2 diabetes mellitus. Similarly, higher serum levels of LDH, creatinine and d-dimer were present in patients who died. Lower $\mathrm{PaO} 2 / \mathrm{FiO} 2$ ratio and absolute lymphocyte count were also most common in these patients. Patients who died were more frequently classified with severe disease at admission, as evaluated by NEWS2 score. There were no differences in the use of tocilizumab and corticosteroids between survivors and non-survivors

A logistic regression analysis was conducted to determine independent factors associated with in-hospital mortality (Table 2). The independent variables associated with in-hospital mortality were age $>65$ years, type 2 diabetes mellitus, NEWS2 $\geqslant 7$ and LDH $>400 \mathrm{U} / \mathrm{L}$. The use of tocilizumab was not related with in-hospital mortality. The Hosmer-Lemeshow test result of the model was 0.598 and the AUROC was 0.84 (CI 95\%, 0.77$0.91)$.

\begin{tabular}{|c|c|c|}
\hline & Adjusted OR (IC95\%) & p-value \\
\hline Age $\geq 65$ years & $6.0(2.4-15.1)$ & $<0.001$ \\
\hline Type 2 diabetes mellitus & $3.0(1.0-9.0)$ & 0.045 \\
\hline NEWS2 $\geq 7$ & $3.5(1.4-8.7)$ & 0.007 \\
\hline $\mathrm{LDH}>400 \mathrm{U} / \mathrm{L}$ & $5.7(2.1-15.2)$ & $<0.001$ \\
\hline Tocilizumab & $0.5(0.1-1.6)$ & 0.273 \\
\hline Corticosteroids & $1.3(0.4-4.3)$ & 0.613 \\
\hline
\end{tabular}

TABLE 2: Logistic regression analysis of factors associated with mortality.

Other variables included in the multivariate model were hypertension, ferritin, d-dimer and c-reactive protein.

NEWS2: National Early Warning Score 2; LDH: lactate dehydrogenase

\section{Subgroup analyses}

Subgroup analyses were performed in patients with hyperinflammatory syndrome and with severe disease. In the multivariate regression analyses, the factors associated with mortality were similar to those in the general population. Tocilizumab was not a protective factor related with in-hospital mortality neither in hyperinflammatory syndrome (OR 0.50 , CI $95 \% 0.15-1.6 ; p=0.25$ ) nor in NEWS $\geqslant 7$ subgroups (OR $0.27, \mathrm{CI}$ $95 \% 0.04-1.9 ; \mathrm{p}=0.19$ ).

\section{Discussion}

In this retrospective cohort study in hospitalized patients with COVID-19, mortality was high and mainly related with older age, type 2 diabetes mellitus, high levels of LDH and the severity of disease at hospital admission. The use of tocilizumab was not related with better prognosis, even in patients with severe disease and patients with hyperinflammatory syndrome.

The present results are consistent with data from studies that evaluated prognostic factors in hospitalized patients with COVID-19. In the univariate analysis of this study, age, type 2 diabetes mellitus, dyspnea, $\mathrm{PaO} / \mathrm{FiO} 2$ ratio, serum levels of d-dimer, $\mathrm{LDH}$ and creatinine, and lymphocyte count were factors associated with a higher risk of in-hospital mortality. In this regard, there is evidence that advanced age, comorbidities, abnormal inflammatory and organ injury circulating biomarkers were more frequently found in patients with an adverse clinical outcome [4]. Thus, clinical history and laboratory profile may help identify patients with a higher risk of in-hospital mortality. In fact, most of these factors have been included in severity scores developed to evaluate the risk of complications in COVID-19 patients [13,14].

We documented a higher mortality among hospitalized COVID-19 patients (39.7\%) compared with that found in other studies (9\%-25\%) [15]. In addition, our cohort also had higher frequency of ICU admission (66.2\%). Factors such as age, sex and comorbidities were not different in our cohort compared with that reported from other COVID-19 patients. However, some factors can explain these poor outcomes in our population. First, Consuegra et al [16] reported the high frequency of early mortality ( $\leqslant 24$ hours of hospital admission) in COVID-19 patients. Early deaths were explained by a more severe disease at hospital admission. Delay in seeking early medical care may be a factor favoring the unacceptably high frequency of mortality during the first 24 hours of hospital admission in these patients. Second, a study documented that 
the risk of dying for COVID-19 among confirmed cases in Colombia was higher in people with subsidized health insurance regime and in people living in very low socioeconomic strata [17]. In this regard, the hospital area of influence, located in the city of Soledad, mainly serves a population with a high level of poverty (78.1\% between strata 1 and 2). Finally, when health system resources were limited, patients with the more severe illness were hospitalized and those with mild illness were transferred to other institutions.

Regarding treatment, studies evaluating interleukin 6 (IL-6) receptor blockade in COVID-19 patients have documented controversial results. Multiple observational studies have shown a discrete mortality reduction in patients with moderate to severe pneumonia due to COVID-19 [18-20]. Similarly, Salama et al. [21] published a multicenter randomized double-blind, placebo-controlled clinical trial performed in patients not receiving mechanical ventilation. Investigators found a reduction in the composite outcome of progression to mechanical ventilation and mortality in those using tocilizumab. Conversely, comparable to our findings, Stone et al. [12] published a randomized double-blind, placebo-controlled clinical trial conducted in patients with COVID-19 pneumonia along with hyperinflammatory syndrome and/or oxygen requirement. This study did not find differences in the mechanical ventilation need or mortality with the use of tocilizumab.

Recently, the REMAP-CAP trial was published reporting a benefit in in-hospital survival and organ supportfree days using tocilizumab and sarilumab [22]. It can be theorized that studies of immunomodulatory treatments have been unsuccessful as a result of the incomplete understanding of the immune response during COVID-19, and the heterogeneity of studies population in terms of age, comorbidities and disease severity. Thus, current guidelines recommend tocilizumab in a specific group of hospitalized adults with progressive severe or critical COVID-19 who have elevated markers of systemic inflammation and who had no response to steroids [23]. Future studies should evaluate if patients have distinct laboratory patterns that reflect various clinical complications requiring different therapy approaches [24].

The strengths of this study are the comprehensive clinical data gathered. This information allowed us to identify specific subgroups of patients. In addition, we performed a multivariate analysis to control confounding factors. The accuracy of the multivariate model was good, as evaluated by the area under the ROC curve. However, our study has some limitations that should be acknowledged. The selection bias restricts the results analysis and the limited sample of patients undermines the power of the study. Moreover, there is no standardized criteria for hyperinflammatory syndrome in COVID-19 and almost every study has a different definition. However, we used the most recent validated COVID-19 hyperinflammatory syndrome criteria in our analysis [5]. Another important limitation was that corticosteroids were not used in all of our patients, because it was not a standard therapy for hospitalized COVID-19 patients during the study period. Finally, some patient features that can be related with outcomes, such as obesity, socioeconomic level, or ethnicity were not recorded.

\section{Conclusions}

In conclusion, COVID-19 mortality was high during the first pandemic wave. The main factors related with mortality were older age, comorbidities as diabetes mellitus, high levels of LDH and the severity of disease at hospital admission according to NEWS2. Tocilizumab had no relation with mortality in global population and in subgroups. Studies should assess if, among COVID-19 patients, there are different inflammatory patterns, such as hyperinflammation, and if different treatment approaches may improve outcomes in specific subgroups of patients.

\section{Additional Information \\ Disclosures}

Human subjects: Consent was obtained or waived by all participants in this study. Comité de Ética en Investigación en el Área de la Salud issued approval ID213-2020. In the Health Science Ethics committee session held on 30th of July 2020, and legalized through act No. 213, the members of the committee unanimously approved the data collection plan of this study. Animal subjects: All authors have confirmed that this study did not involve animal subjects or tissue. Conflicts of interest: In compliance with the ICMJE uniform disclosure form, all authors declare the following: Payment/services info: All authors have declared that no financial support was received from any organization for the submitted work. Financial relationships: All authors have declared that they have no financial relationships at present or within the previous three years with any organizations that might have an interest in the submitted work. Other relationships: All authors have declared that there are no other relationships or activities that could appear to have influenced the submitted work.

\section{Acknowledgements}

The authors wish to thank Universidad del Norte and Hospital Universidad del Norte for supporting this project

\section{References}

1. World Health Organization. (2020). Accessed: May 20, 2020: http://://www.who.int/docs/defaultsource/coronaviruse/situation-reports/20200311-sitrep-51-covid19.pdf?sfvrsn=1ba62e.... 
2. Søvik S, Bådstøløkken PM, Sørensen V, Myhre PL, Prebensen C, Omland T, Berdal JE: A single-centre, prospective cohort study of COVID-19 patients admitted to ICU for mechanical ventilatory support. Acta Anaesthesiol Scand. 2021, 65:351-9. 10.1111/aas.13726

3. Armstrong RA, Kane AD, Cook TM: Outcomes from intensive care in patients with COVID-19: a systematic review and meta-analysis of observational studies. Anaesthesia. 2020, 75:1340-9. 10.1111/anae.15201

4. Figliozzi S, Masci PG, Ahmadi N, et al.: Predictors of adverse prognosis in COVID-19: A systematic review and meta-analysis. Eur J Clin Invest. 2020, 50:e13362. 10.1111/eci.13362

5. Webb BJ, Peltan ID, Jensen P, et al.: Clinical criteria for COVID-19-associated hyperinflammatory syndrome: a cohort study. Lancet Rheumatol. 2020, 2:e754-63. 10.1016/S2665-9913(20)30343-X

6. Horby P, Lim WS, Emberson JR, et al.: Dexamethasone in hospitalized patients with Covid-19. N Engl J Med. 2021, 384:693-704. 10.1056/NEJMoa2021436

7. Colaneri M, Bogliolo L, Valsecchi P, et al.: Tocilizumab for Treatment of Severe COVID-19 Patients: Preliminary Results from SMAtteo COvid19 REgistry (SMACORE). Microorganisms. 2020, 8:10.3390/microorganisms8050695

8. Klopfenstein T, Zayet S, Lohse A, et al.: Tocilizumab therapy reduced intensive care unit admissions and/or mortality in COVID-19 patients. Med Mal Infect. 2020, 50:397-400. 10.1016/j.medmal.2020.05.001

9. Moreno-García E, Rico V, Albiach L, et al.: Tocilizumab is associated with reduced risk of ICU admission and mortality in patients with SARS-CoV-2 infection. medRxiv. 2020, 1:e1. 10.1101/2020.06.05.20113738

10. Luo P, Liu Y, Qiu L, Liu X, Liu D, Li J: Tocilizumab treatment in COVID-19: a single center experience . J Med Virol. 2020, 92:814-8. 10.1002/jmv.25801

11. Salvarani C, Dolci G, Massari M, et al.: Effect of tocilizumab vs standard care on clinical worsening in patients hospitalized with COVID-19 pneumonia: a randomized clinical trial. JAMA Intern Med. 2021, 181:24-31. 10.1001/jamainternmed.2020.6615

12. Stone JH, Frigault MJ, Serling-Boyd NJ, et al.: Efficacy of tocilizumab in patients hospitalized with Covid-19 . N Engl J Med. 2020, 383:2333-44. 10.1056/NEJMoa2028836

13. Myrstad M, Ihle-Hansen H, Tveita AA, Andersen EL, Nygård S, Tveit A, Berge T: National Early Warning Score 2 (NEWS2) on admission predicts severe disease and in-hospital mortality from Covid-19 - a prospective cohort study. Scand J Trauma Resusc Emerg Med. 2020, 28:66. 10.1186/s13049-020-00764-3

14. Liang W, Liang H, Ou L, et al.: Development and validation of a clinical risk score to predict the occurrence of critical illness in hospitalized patients with COVID-19. JAMA Intern Med. 2020, 180:1081-9. 10.1001/jamainternmed.2020.2033

15. Asch DA, Sheils NE, Islam MN, Chen Y, Werner RM, Buresh J, Doshi JA: Variation in US hospital mortality rates for patients admitted with COVID-19 during the first 6 months of the pandemic. JAMA Intern Med. 2021, 181:471-8. 10.1001/jamainternmed.2020.8193

16. Consuegra D, Seidner-Isaacs Y, Larios-Sanjuan D, et al.: Unexpected high frequency of early mortality in COVID-19: a single-centre experience during the first wave of the pandemic. Intern Med J. 2021, 51:102-5. 10.1111/imj.15134

17. Cifuentes MP, Rodriguez-Villamizar LA, Rojas-Botero ML, et al.: Socioeconomic inequalities associated with mortality for COVID-19 in Colombia: a cohort nationwide study. J Epidemiol Community Health. 2021, 1:e216275. 10.1101/2020.12.14.20248203

18. Somers EC, Eschenauer GA, Troost JP, et al.: Tocilizumab for treatment of mechanically ventilated patients with COVID-19. medRxiv. 2020, 10.1101/2020.05.29.20117358

19. Rossotti R, Travi G, Ughi N, et al.: Safety and efficacy of anti-il6-receptor tocilizumab use in severe and critical patients affected by coronavirus disease 2019: a comparative analysis. J Infect. 2020, 81:e11-7. 10.1016/j.jinf.2020.07.008

20. Potere N, Di Nisio M, Rizzo G, et al.: Low-dose subcutaneous tocilizumab to prevent disease progression in patients with moderate COVID-19 pneumonia and hyperinflammation. Int J Infect Dis. 2020, 100:421-4. 10.1016/j.ijid.2020.07.078

21. Salama C, Han J, Yau L, et al.: Tocilizumab in patients hospitalized with Covid-19 pneumonia . N Engl J Med. 2021, 384:20-30. 10.1056/NEJMoa2030340

22. Gordon AC, Mouncey PR, Al-Beidh F, et al.: Interleukin-6 receptor antagonists in critically ill patients with Covid-19. N Engl J Med. 2021, 384:1491-502. 10.1056/NEJMoa2100433

23. IDSA Guidelines on the Treatment and Management of Patients with COVID-19 . (2021). Accessed: March 18: http://www.idsociety.org/practice-guideline/covid-19-guideline-treatment-and-management.

24. Garcia-Vidal C, Moreno-García E, Hernández-Meneses M, et al.: Personalized therapy approach for hospitalized patients with COVID-19. Clin Infect Dis. 2020, 10.1093/cid/ciaa964 